\title{
Closed-form algorithms for computing the intersection of two subspaces
}

\author{
YAN Fenggang, LIU Shuai, WANG Jun, and JIN Ming* \\ School of Information Science and Engineering, Harbin Institute of Technology at Weihai, Weihai 264209, China
}

\begin{abstract}
Finding the intersection of two subspaces is of great interest in many fields of signal processing. Over several decades, there have been numerous formulas discovered to solve this problem, among which the alternate projection method (APM) is the most popular one. However, APM suffers from high computational complexity, especially for real-time applications. Moreover, APM only gives the projection instead of the orthogonal basis of two given subspaces. This paper presents two alternate algorithms which have a closed form and reduced complexity as compared to the APM technique. Numerical simulations are conducted to verify the correctness and the effectiveness of the proposed methods.
\end{abstract}

Keywords: orthogonal projection, singular value decomposition, alternate projection method (APM), intersection.

DOI: $10.21629 /$ JSEE.2019.02.03

\section{Introduction}

It is a fundamental task of great interest to find the intersection of two subspaces, which has been widely used in many applications in various fields. For example, in array signal processing including beam-forming [1] and source localization [2], there is generally a need for the determination of the direction of arrivals (DOAs) of multiple signals impinging on an antenna array [3]. As most DOA estimation algorithms are based on the orthogonality between the so-called signal- and noise- subspaces [4], computation of sub-space intersection is extensively required by various DOA estimators $[5,6]$. Such direction estimation technique with subspace intersection computation is also widely required to solve the problem of bearing estimation in shallow ocean by using acoustic vector sensors [7-9]. Another example is the wireless communication system, where computation of subspace intersection is often used

Manuscript received February 22, 2017.

*Corresponding author.

This work was supported by the National Natural Science Foundation of China (61501142; 61871149), and the project supported by Discipline Construction Guiding Foundation in Harbin Institute of Technology (Weihai) (WH2-0160107). to solve the asynchronous co-channel interference mitigation problem [10], and to estimate parameters such as multicomponent polynomial-phase signals [11] and blind time delay [12]. Besides, computation of subspace intersection is also used in many other areas such as subspace learning for computer vision [13], multicell scheduling for coordinated multiple-input multiple-output (MIMO) cellular networks [14], MIMO radar [15], computation of specular reflections in complex 3D enclosures [16], and so on.

\section{Definition and literature review}

For the sake of notation simplicity, we use $\mathbf{C}^{m \times n}$ to denote the set of $m \times n$ complex matrices, and use $\operatorname{span}(\boldsymbol{A})$ and $\operatorname{null}(\boldsymbol{A})$ to stand for the column- and null- spaces of the matrix $\boldsymbol{A} \in \mathbf{C}^{m \times n}$, respectively, which are given by

$$
\begin{gathered}
\operatorname{span}(\boldsymbol{A}) \triangleq\left\{\boldsymbol{x} \in \mathbf{C}^{m \times 1} \mid \boldsymbol{x}=\sum_{i-1}^{n} k_{i} \boldsymbol{a}_{i}\right\} \\
\operatorname{null}(\boldsymbol{A}) \triangleq\left\{\boldsymbol{x} \in \mathbf{C}^{m \times 1} \mid \boldsymbol{A} \boldsymbol{x}=0\right\}
\end{gathered}
$$

respectively, where $\boldsymbol{a}_{i}$ is the $i$ th column of $\boldsymbol{A}$ and $k_{i}$ is an unknown scalar. In addition, let $\boldsymbol{\Psi}_{i} \in \mathbf{C}^{m \times r_{i}}$ be the orthogonal basis of two subspaces $\operatorname{span}\left(\boldsymbol{\Psi}_{i}\right), m>r_{i}(i=$ $1,2)$, we use

$$
\boldsymbol{P}_{i} \triangleq \boldsymbol{\Psi}_{i} \boldsymbol{\Psi}_{i}^{\mathrm{H}}, \quad i=1,2
$$

to denote the projection matrices of the two subspaces, respectively. The intersection of two subspaces $\operatorname{span}\left(\boldsymbol{\Psi}_{i}\right)(i=1,2)$ is defined as

$$
\operatorname{span}(\boldsymbol{\Psi}) \triangleq \operatorname{span}\left(\boldsymbol{\Psi}_{1}\right) \cap \operatorname{span}\left(\boldsymbol{\Psi}_{2}\right)
$$

where $\boldsymbol{\Psi}$ is the orthogonal basis of $\operatorname{span}(\boldsymbol{\Psi})$, and the projection matrix of the intersection is given by

$$
\boldsymbol{P} \triangleq \Psi \boldsymbol{\Psi}
$$

For the vector $\boldsymbol{x}$ and the matrix $\boldsymbol{A},\|\boldsymbol{x}\|$ and $\|\boldsymbol{A}\|_{\mathrm{F}}$ stand for the Euclidean norm of $\boldsymbol{x}$ and the Euclidean norm of $\boldsymbol{A}$, respectively. In addition, $\boldsymbol{A}^{\mathrm{H}}$ stands for Hermitian transpose or just transpose if $\boldsymbol{A}$ is real. 
Over several decades, there have been numerous formulas discovered to compute intersection of two subspaces. Here, we briefly summarize some representative works in this field. In [17], Golub and van Loan suggested to compute $\boldsymbol{\Psi}$ by using the singular value decomposition (SVD) of multiplication of $\boldsymbol{\Psi}_{1}$ and $\boldsymbol{\Psi}_{2}$

$$
\boldsymbol{M} \triangleq \boldsymbol{\Psi}_{1}^{\mathrm{H}} \boldsymbol{\Psi}_{2}=\boldsymbol{U}^{\mathrm{H}} \prod V
$$

as follows:

$$
\boldsymbol{\Psi}=\boldsymbol{\Psi}_{1} \boldsymbol{U}\left(:, 1: m-r_{1}-r_{2}\right)
$$

where $\boldsymbol{\Psi}=\boldsymbol{\Psi}_{1} \boldsymbol{U}\left(:, 1: m-r_{1}-r_{2}\right)$ denotes the sub-matrix composed of the columns of $\boldsymbol{\Psi}_{1} \boldsymbol{U}$ from 1 to $m-r_{1}-r_{2}$. In [18], Anderson and Duffin gave an explicit and closed-form formula for computing $\boldsymbol{P}$ as

$$
\boldsymbol{P}=2 \boldsymbol{P}_{1}\left(\boldsymbol{P}_{1}+\boldsymbol{P}_{2}\right)^{\dagger} \boldsymbol{P}_{2}
$$

where $\dagger$ denotes the Moore-Penrose inverse. Besides the above two algorithms, the alternate projection method (APM) [19-25] offers a more popular solution to compute the intersection. In [19], von Neumann gave the projection $\boldsymbol{P}$ as the limit

$$
\boldsymbol{P}=\lim _{n \rightarrow \infty}\left(\boldsymbol{P}_{1} \boldsymbol{P}_{2}\right)^{n}
$$

The convergence rate of (9) was established by Aronszajn [20] and Kayalar [21] as

$$
\left\|\left(\boldsymbol{P}_{1} \boldsymbol{P}_{2}\right)^{n}-\boldsymbol{P}\right\|_{\mathrm{F}} \leqslant c^{2 n-1}
$$

where

$$
c=\sup \left\{\begin{aligned}
\frac{\langle\boldsymbol{x}, \boldsymbol{y}\rangle}{\|\boldsymbol{x}\|\|\boldsymbol{y}\|} \mid \boldsymbol{x} & \in \operatorname{span}\left(\boldsymbol{\Psi}_{1}\right) \cap[\operatorname{span}(\boldsymbol{\Psi})]^{\perp} \\
\boldsymbol{y} & \in \operatorname{span}\left(\boldsymbol{\Psi}_{2}\right) \cap[\operatorname{span}(\boldsymbol{\Psi})]^{\perp}
\end{aligned}\right\}
$$

is the cosine of the minimal angle between $\operatorname{span}\left(\boldsymbol{\Psi}_{1}\right) \cap$ $[\operatorname{span}(\boldsymbol{\Psi})]^{\perp}$ and $\operatorname{span}\left(\boldsymbol{\Psi}_{2}\right) \cap[\operatorname{span}(\boldsymbol{\Psi})]^{\perp}[22]$. A similar bound for the convergence rate of (9) was given by Deutsch in [23]. These ideas of APM were extended by Halperin to the case of more than two subspaces in [24], and were addressed and compared by Ben-Israel in [25]. More recently, Zhou [26] developed an improved URV decomposition method to solve the asyn-chronous cochannel interference mitigation problem in cellular systems. Zhang [27] proposed an APM-based method to estimate the desired signal subspace by the intersection between the signal-plus-interference subspaces, to enhance the robustness of adaptive beamforming.
On the other hand, considerable efforts have focused on understanding and analyzing performance and convergence rates of the APM algorithm. In [28,29], convex and non-convex feasibility problems were analyzed.

In [30-32], convex optimization and monotone inclusion problems were addressed. For feasibility problems with two subspaces, it has been well known that the standard APM converges linearly with exact rate being the squared cosine of the Friedrichs angle [22]. Recently reported results in [33] demonstrate that the DouglasRachford algorithm [34] converges with a rate given by the Friedrichs angle. In addition, Bauschke [35] provided sharp convergence rates for the APM algorithm and applied the result to find optimal parameters for the generalized alternating projections method. Fält and Giselsson [36] extended the results of [35] and optimized the sharp convergence rate for the generalized APM.

Despite its interesting contributions, it is seen from (9) that the famous APM algorithm has a high computational complexity because it involves an iteration of the matrix product $\left(\boldsymbol{P}_{1} \boldsymbol{P}_{2}\right)^{n}$. Moreover, APM only gives the orthogonal projection onto the intersection of two subspaces instead of the orthogonal basis of their intersection. Although (7) and (8) have lower complexities than APM, they still involve multiple matrix multiplication computations.

\section{Proposed m-SVD and s-SVD algorithms}

To overcome the above mentioned drawbacks and limits, we develop two new methods which can directly obtain the orthogonal basis $\boldsymbol{\Psi}$ with reduced complexity as compared to state-of-the-art methods including APM, (7) and (8). The two methods start by constructing two matrices $\boldsymbol{A}$ and $\boldsymbol{B}$ by using the multiplication- and sum- of $\boldsymbol{P}_{1}$ and $\boldsymbol{P}_{2}$ as

$$
\begin{gathered}
\boldsymbol{A} \triangleq \boldsymbol{I}-\boldsymbol{P}_{1} \boldsymbol{P}_{2} \\
\boldsymbol{B} \triangleq 2 \boldsymbol{I}-\boldsymbol{P}_{1}-\boldsymbol{P}_{2}
\end{gathered}
$$

and preform singular value decomposition (SVD) on $\boldsymbol{A}$ and $\boldsymbol{B}$ to get $\boldsymbol{\Psi}$. Therefore, the two methods are referred to respectively as the multiplication-SVD (m-SVD)- and the sum-SVD (s-SVD)- algorithms in this paper.

The theoretical fundamentation of the proposed mSVD- and s-SVD- algorithms are given by the following two theorems, respectively.

Theorem 1 Let $\boldsymbol{A}$ be defined by (12), then we have

$$
\operatorname{span}(\boldsymbol{\Psi})=\operatorname{null}(\boldsymbol{A}) .
$$

Proof Let $\boldsymbol{x} \in \operatorname{span}(\boldsymbol{\Psi})$, then we must have $\boldsymbol{x} \in$ $\operatorname{span}\left(\boldsymbol{\Psi}_{1}\right)$ and $\boldsymbol{x} \in \operatorname{span}\left(\boldsymbol{\Psi}_{2}\right)$. Since $\boldsymbol{P}_{1}$ and $\boldsymbol{P}_{2}$ are the projection matrices of $\operatorname{span}\left(\boldsymbol{\Psi}_{1}\right)$ and $\operatorname{span}\left(\boldsymbol{\Psi}_{2}\right)$, respectively, we further have $\boldsymbol{x}=\boldsymbol{P}_{1} \boldsymbol{x}$ and $\boldsymbol{x}=\boldsymbol{P}_{2} \boldsymbol{x}$. 
Therefore, we obtain $\boldsymbol{x}=\boldsymbol{P}_{1} \boldsymbol{P}_{2} \boldsymbol{x}$, which is equivalent to $\boldsymbol{A} \boldsymbol{x}=\left(\boldsymbol{I}-\boldsymbol{P}_{1} \boldsymbol{P}_{2}\right) \boldsymbol{x}=\boldsymbol{x}-\boldsymbol{P}_{1} \boldsymbol{P}_{2} \boldsymbol{x}=0$. This implies that $\boldsymbol{x} \in \operatorname{null}(\boldsymbol{A})$, and we have

$$
\operatorname{span}(\boldsymbol{\Psi}) \subseteq \operatorname{null}(\boldsymbol{A}) .
$$

On the other hand, let $\boldsymbol{x} \in \operatorname{null}(\boldsymbol{A})$, then we must have $\boldsymbol{A} \boldsymbol{x}=0$, which implies $\boldsymbol{x}=\boldsymbol{P}_{1} \boldsymbol{P}_{2} \boldsymbol{x}$. Noting that $\boldsymbol{P}_{1} \boldsymbol{P}_{2} \boldsymbol{x}$ is the projection of $\boldsymbol{P}_{2} \boldsymbol{x}$ onto subspace $\operatorname{span}\left(\boldsymbol{\Psi}_{1}\right)$ and $\boldsymbol{P}_{2} \boldsymbol{x}$ is the projection of $\boldsymbol{x}$ onto subspace $\operatorname{span}\left(\boldsymbol{\Psi}_{2}\right)$, we can obtain $\|\boldsymbol{x}\|^{2}=\left\|\boldsymbol{P}_{1} \boldsymbol{P}_{2} \boldsymbol{x}\right\|^{2} \leqslant\left\|\boldsymbol{P}_{2} \boldsymbol{x}\right\|^{2} \leqslant\|\boldsymbol{x}\|^{2}$. It follows directly from the above inequations that $\boldsymbol{x}=\boldsymbol{P}_{2} \boldsymbol{x}$, which indicates that $\boldsymbol{x} \in \operatorname{span}\left(\boldsymbol{\Psi}_{2}\right)$. Substituting $\boldsymbol{x}=\boldsymbol{P}_{2} \boldsymbol{x}$ into $\boldsymbol{x}=\boldsymbol{P}_{1} \boldsymbol{P}_{2} \boldsymbol{x}$ leads to $\boldsymbol{x}=\boldsymbol{P}_{1} \boldsymbol{x}$, and we also have $\boldsymbol{x} \in \operatorname{span}\left(\boldsymbol{\Psi}_{1}\right)$. Therefore, we have $\boldsymbol{x} \in \operatorname{span}(\boldsymbol{\Psi})$, which is equivalent to

$$
\operatorname{span}(\boldsymbol{A}) \subseteq \operatorname{null}(\boldsymbol{\Psi}) .
$$

Combining (15) and (16) leads to $\operatorname{span}(\boldsymbol{A})=\operatorname{null}(\boldsymbol{\Psi})$.

Theorem 2 Let $B$ defined by (13), then we have

$$
\operatorname{span}(\boldsymbol{\Psi})=\operatorname{null}(\boldsymbol{B}) .
$$

Proof Suppose that $\boldsymbol{x} \in \operatorname{span}(\boldsymbol{\Psi})$, we must have $\boldsymbol{x} \in \operatorname{span}\left(\boldsymbol{\Psi}_{1}\right)$ and $\boldsymbol{x} \in \operatorname{span}\left(\boldsymbol{\Psi}_{1}\right)$. Since $\boldsymbol{P}_{i}(i=1,2)$ is the projection matrix of $\operatorname{span}\left(\boldsymbol{\Psi}_{i}\right)(i=1,2)$, we have $\boldsymbol{x}=\boldsymbol{x}=\boldsymbol{P}_{2} \boldsymbol{x}$, which means $\boldsymbol{B} \boldsymbol{x}=\left(\boldsymbol{I}-\boldsymbol{P}_{1} \boldsymbol{P}_{2}\right) \boldsymbol{x}=$ $\boldsymbol{x}-\boldsymbol{P}_{1} \boldsymbol{P}_{2} \boldsymbol{x}=0$. It follows directly from the above equation that $\boldsymbol{x} \in \operatorname{null}(\boldsymbol{B})$, and therefore we obtain

$$
\operatorname{span}(\boldsymbol{\Psi}) \subseteq \operatorname{null}(\boldsymbol{B}) .
$$

On the other hand, assume that $\boldsymbol{x} \in \operatorname{null}(\boldsymbol{B})$, then we must have $\boldsymbol{B} \boldsymbol{x}=0$, which is equivalent to $2 \boldsymbol{x}=$ $\boldsymbol{P}_{1} \boldsymbol{x}+\boldsymbol{P}_{2} \boldsymbol{x}$. Take norm on both sides of the above equation leads to $2\|\boldsymbol{x}\|=\left\|\boldsymbol{P}_{1} \boldsymbol{x}+\boldsymbol{P}_{2} \boldsymbol{x}\right\| \leqslant\left\|\boldsymbol{P}_{1} \boldsymbol{x}\right\|+\left\|\boldsymbol{P}_{2} \boldsymbol{x}\right\|$. Noting that $\boldsymbol{P}_{i} \boldsymbol{x}(i=1,2)$ is the projection of $\boldsymbol{x}$ onto subspace $\boldsymbol{\Psi}_{i}(i=1,2)$, we have $\left\|\boldsymbol{P}_{1} \boldsymbol{x}\right\| \leqslant\|\boldsymbol{x}\|$ and $\left\|\boldsymbol{P}_{2} \boldsymbol{x}\right\| \leqslant\|\boldsymbol{x}\|$. Therefore, we further obtain $2\|\boldsymbol{x}\| \leqslant\left\|\boldsymbol{P}_{1} \boldsymbol{x}\right\|+\left\|\boldsymbol{P}_{2} \boldsymbol{x}\right\| \leqslant$ $2\|\boldsymbol{x}\|$. It follows that $\left\|\boldsymbol{P}_{1} \boldsymbol{x}\right\|=\left\|\boldsymbol{P}_{2} \boldsymbol{x}\right\|=\|\boldsymbol{x}\|$, which indicates that $\boldsymbol{x}=\boldsymbol{P}_{1} \boldsymbol{x} \in \operatorname{span}\left(\boldsymbol{\Psi}_{1}\right), \boldsymbol{x}=\boldsymbol{P}_{2} \boldsymbol{x} \in \operatorname{span}\left(\boldsymbol{\Psi}_{2}\right)$. Hence, $\boldsymbol{x} \in \operatorname{span}(\boldsymbol{\Psi})$, and we obtain

$$
\operatorname{span}(\boldsymbol{B}) \subseteq \operatorname{null}(\boldsymbol{\Psi})
$$

Combining (18) and (19) leads to $\operatorname{span}(\boldsymbol{\Psi}) \subseteq \operatorname{null}(\boldsymbol{B})$.

Now, we compute $\boldsymbol{\Psi}$ by exploiting the SVDs of $\boldsymbol{A}$ and $\boldsymbol{B}$. Let $\operatorname{dim}[\operatorname{span}(\cdot)]$ stand for the dimension of the embraced subspace, we have $\operatorname{rank}(\boldsymbol{A})=\operatorname{rank}(\boldsymbol{B})=$ $\operatorname{dim}[\operatorname{span}(\boldsymbol{\Psi})]=r$. Hence, the SVDs of $\boldsymbol{A}$ and $\boldsymbol{B}$ can be expressed as

$$
\boldsymbol{A}=\sum_{i=1}^{r} \sigma_{i} \boldsymbol{u}_{i} \boldsymbol{v}_{i}^{\mathrm{H}}
$$

$$
\boldsymbol{B}=\sum_{j=1}^{r} \sigma_{j} \boldsymbol{p}_{j} \boldsymbol{q}_{j}^{\mathrm{H}} .
$$

Using $\boldsymbol{v}_{i}^{\mathrm{H}} \boldsymbol{v}_{j}=0, \boldsymbol{q}_{i} \boldsymbol{q}_{j}^{\mathrm{H}}=0, i \neq j$, we have

$$
\boldsymbol{A} \boldsymbol{v}_{j}=\boldsymbol{B} \boldsymbol{q}_{j}=0, \quad \forall j=r+1 ; r+2, \ldots, m .
$$

Therefore, we have

$$
\begin{aligned}
& \operatorname{null}(\boldsymbol{A})=\operatorname{span}\left(\boldsymbol{v}_{r+1}, \boldsymbol{v}_{r+2}, \ldots, \boldsymbol{v}_{m}\right) \\
& \operatorname{null}(\boldsymbol{B})=\operatorname{span}\left(\boldsymbol{q}_{r+1}, \boldsymbol{q}_{r+2}, \ldots, \boldsymbol{q}_{m}\right) .
\end{aligned}
$$

Combining (14), (17), (23) and (24), $\boldsymbol{\Psi}$ is given by

$$
\boldsymbol{\Psi}=\left[\boldsymbol{v}_{r+1}, \boldsymbol{v}_{r+2}, \ldots, \boldsymbol{v}_{m}\right]
$$

or equally given by

$$
\boldsymbol{\Psi}=\left[\boldsymbol{q}_{r+1}, \boldsymbol{q}_{r+2}, \ldots, \boldsymbol{q}_{m}\right] .
$$

\section{Summary and complexity analysis}

Detailed steps for implementing the proposed m-SVD and s-SVD algorithms are summarized in Algorithm 1 and Algorithm 2, respectively.

Algorithm 1 Proposed m-SVD algorithm

Input: $\boldsymbol{\Psi}_{1}, \boldsymbol{\Psi}_{2} \rightarrow$ Orthogonal bases of two subspaces

Output: $\boldsymbol{\Psi} \rightarrow$ Orthogonal basis of the intersection

i. Compute the two projections $\boldsymbol{P}_{i} \triangleq \boldsymbol{\Psi}_{i} \boldsymbol{\Psi}_{i}^{\mathrm{H}}(i=1,2)$;

ii. Compute matrix $\boldsymbol{A}$ using $\boldsymbol{P}_{1}$ and $\boldsymbol{P}_{2}$ by (12);

iii. Perform SVD on $\boldsymbol{A}$ according to (20);

iv. Obtain $\boldsymbol{\Psi}$ by (25).

Algorithm 2 Proposed s-SVD algorithm

Input: $\boldsymbol{\Psi}_{1}, \boldsymbol{\Psi}_{2} \rightarrow$ Orthogonal bases of two subspaces

Output: $\boldsymbol{\Psi} \rightarrow$ Orthogonal basis of the intersection

i. Compute the two projections $\boldsymbol{P}_{i} \triangleq \boldsymbol{\Psi}_{i} \boldsymbol{\Psi}_{i}^{\mathrm{H}}(i=1,2)$;

ii. Compute matrix $\boldsymbol{B}$ using $\boldsymbol{P}_{1}$ and $\boldsymbol{P}_{2}$ by (13);

iii. Perform SVD on $\boldsymbol{B}$ according to (21);

iv. Obtain $\boldsymbol{\Psi}$ by (26).

Primary computational flops required by APM, (7), (8) and the proposed $\mathrm{m}-\mathrm{SVD}$ and s-SVD algorithms are compared in Table 1 . As $\boldsymbol{\Psi}_{i} \in \mathbf{C}$, compute $\boldsymbol{P}_{i}$ costs $O\left(m^{2} r_{i}\right)$ flops $(i=1,2)$. Note that the dimensions of $\boldsymbol{P}_{1}$ and $\boldsymbol{P}_{2}$ are both $m \times m$, both the product $\boldsymbol{P}_{1} \boldsymbol{P}_{2}$ and the MoorePenrose inverse $\left(\boldsymbol{P}_{1}+\boldsymbol{P}_{2}\right)^{\dagger}$ cost $O\left(m^{3}\right)$ flops. Since both $\boldsymbol{A}$ and $\boldsymbol{B}$ are of $\operatorname{rank}(r)$, the SVD of $\boldsymbol{A}$ and that of $\boldsymbol{B}$ costs $O\left(m^{2} r_{i}\right)$ flops [17].

Table 1 Comparison of computational flops

\begin{tabular}{cc}
\hline Algorithm & Primary computational flops \\
\hline APM & $O\left(m^{3 n}\right)$ \\
Method in (7) & $O\left[m^{3}+m r_{1} r_{2}+m r_{1}\left(m-r_{1}-r_{2}\right)\right]$ \\
Method in (8) & $O\left(3 m^{3}+m^{2} r_{1}+m^{2} r_{2}\right)$ \\
m-SVD & $O\left(m^{3}+m+m^{2} r+m^{2} r_{1}+m^{2} r_{2}\right)$ \\
s-SVM & $O\left(2 m+m^{2} r+m^{2} r_{1}+m^{2} r_{2}\right)$ \\
\hline
\end{tabular}


It is to be shown in the simulation part that APM generally requires $n \gg 1$ to achieve acceptable accuracy. Therefore, it can be concluded from Table 1 that the proposed $\mathrm{m}-\mathrm{SVD}$ - and s-SVD- methods save a significant computational complexity as compared to APM. On the other hand, both (7) and (8) involve the cube of $m$, and the two methods cost higher complexity than the proposed algorithms, especially when $m$ is a big number.

\section{Simulation results}

Numerical simulations are conducted to verify the effectiveness of the proposed methods and compare it to APM and the two techniques in (7) and (8). In the simulation, we set $m=15, r_{1}=r_{2}=11$, and let $\boldsymbol{\Psi}_{i} \in \mathbf{C}^{m \times r_{i}}(i=1,2)$ be randomly generated in the Matlab 7.0 environment by

$$
\begin{gathered}
\boldsymbol{\Psi}_{i}=\operatorname{Schmidt}\left(\boldsymbol{T}_{i}\right) \\
\boldsymbol{T}_{i}=\operatorname{randn}\left(m, r_{i}\right)+\mathrm{j} * \operatorname{randn}\left(m, r_{i}\right)
\end{gathered}
$$

to guarantee $\boldsymbol{\Psi}_{i} \boldsymbol{\Psi}_{i}^{\mathrm{H}}=\boldsymbol{P}_{i}$ and $\boldsymbol{\Psi}_{i}^{\mathrm{H}} \boldsymbol{\Psi}_{i}=\boldsymbol{I}$, where Schmidt( $(\cdot)$ is Gram-Schmidt orthonormalization.

To verify the effectiveness of the proposed methods and compare it to APM and the two methods in (7) and (8), we define

$$
\begin{gathered}
d_{1}=\left\|\boldsymbol{P}_{\mathrm{m}-\mathrm{SVD}}-\boldsymbol{P}^{(7)}\right\|_{\mathrm{F}} \\
d_{2}=\left\|\boldsymbol{P}_{\mathrm{m}-\mathrm{SVD}}-\boldsymbol{P}^{(8)}\right\|_{\mathrm{F}} \\
d_{3}=\left\|\boldsymbol{P}_{\mathrm{s}-\mathrm{SVD}}-\boldsymbol{P}^{(7)}\right\|_{\mathrm{F}} \\
d_{4}=\left\|\boldsymbol{P}_{\mathrm{s}-\mathrm{SVD}}-\boldsymbol{P}^{(8)}\right\|_{\mathrm{F}} \\
d_{5}=\left\|\boldsymbol{P}_{\mathrm{m}-\mathrm{SVD}}-\boldsymbol{P}_{n}^{\mathrm{APM}}\right\|_{\mathrm{F}}^{2} \\
d_{6}=\left\|\boldsymbol{P}_{\mathrm{s}-\mathrm{SVD}}-\boldsymbol{P}_{n}^{\mathrm{APM}}\right\|_{\mathrm{F}}^{2} \\
d_{7}=\left\|\boldsymbol{P}_{\mathrm{m}-\mathrm{SVD}}-\boldsymbol{P}_{\mathrm{s}-\mathrm{SVD}}\right\|_{\mathrm{F}}^{2}
\end{gathered}
$$

where $\boldsymbol{P}_{\mathrm{m}-\mathrm{SVD}}$ and $\boldsymbol{P}_{\mathrm{s}-\mathrm{SVD}}$ are the projection matrices of intersection computed by the proposed m-SVD- and sSVD- methods, respectively. $\boldsymbol{P}^{(7)}$ and $\boldsymbol{P}^{(8)}$ stand for the projection matrices of intersection computed by (7) and (8), respectively. $\boldsymbol{P}_{n}^{\mathrm{APM}}$ denotes the projection matrix of intersection computed by APM with iteration number $n$. Thus, $d_{i}(i=1, \ldots, 7)$ can be used to describe the distances among $\boldsymbol{P}_{\mathrm{m}-\mathrm{SVD}}, \boldsymbol{P}_{\mathrm{s}-\mathrm{SVD}}, \boldsymbol{P}^{(7)}, \boldsymbol{P}^{(8)}$ and $\boldsymbol{P}_{n}^{\mathrm{APM}}$, which can be also used to compare the accuracies among the five algorithms.

First, we examine the accuracy of the proposed methods and compare it to state-of-the-art techniques. We plot $d_{i}(i=1, \ldots, 7)$ in Fig. 1 and Fig. 2 as functions of the iteration number $n$, where $n$ varies over a wide range from $n=1$ to $n=100$. It is seen clearly from the two figures that for $n=1$ to $n=50, d_{7} \equiv 0$ and $d_{i} \approx 0(i=1,2,3,4)$. This implies that the proposed $\mathrm{m}$ SVD- and s-SVD- methods provide exactly the same results as those by (7) and (8), which verifies the theoretical analysis in Theorem 1 and Theorem 2. It is also seen from the two figures that the values of $d_{5}$ and $d_{6}$ decrease dramatically as $n$ increases. This indicates that APM converges to the proposed $\mathrm{m}-\mathrm{SVD}$ - and s-SVD- algorithms as $n$ increases. Only for sufficiently large $n$ ( $n \geqslant 10$ as shown in the two figures), APM achieves comparable accuracy as compared to the proposed m-SVD- and s-SVD- methods. Therefore, the proposed methods show reduced complexity with improved accuracy as compared to APM.

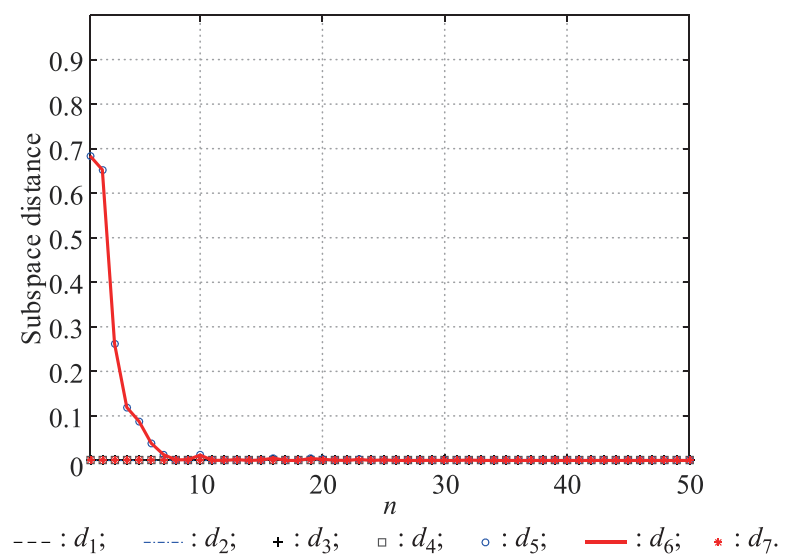

Fig. 1 Subspace distance versus iteration number $n$, axis $Y$ denoted in linear scale

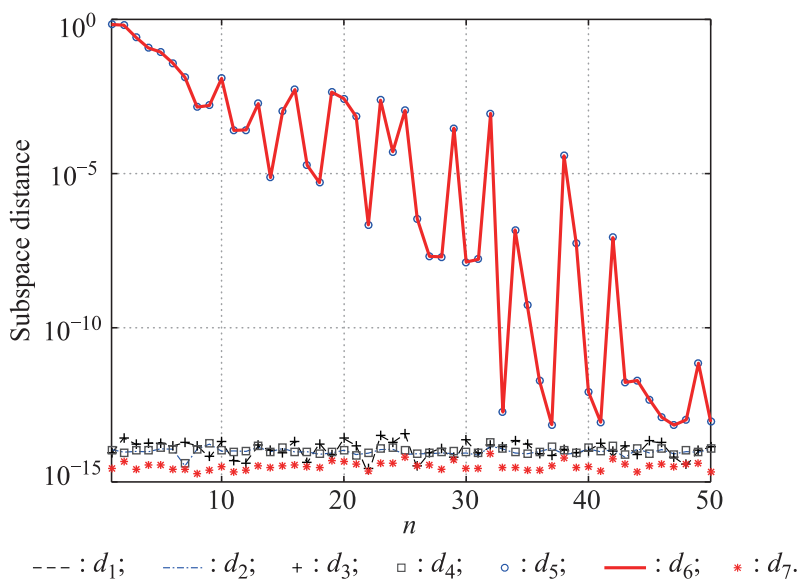

Fig. 2 Subspace distance versus iteration number $n$, axis $Y$ denoted in log scale

Next, we investigate the proposed methods with an example of practical scenario where the proposed techniques could be applied. We consider the DOA estimation problem by using the multiple signal classification (MUSIC) algorithm [3-6]. We use the proposed methods and APM with $n=7$ to compute the intersection of the noise subspace. We compare different MUSIC spectrums constructed by using the proposed methods and APM in Fig. 3. Note that m-SVD and s-SVD provide exactly the 
same accuracy, the spectrums constructed by m-SVD overlap those by s-SVD.

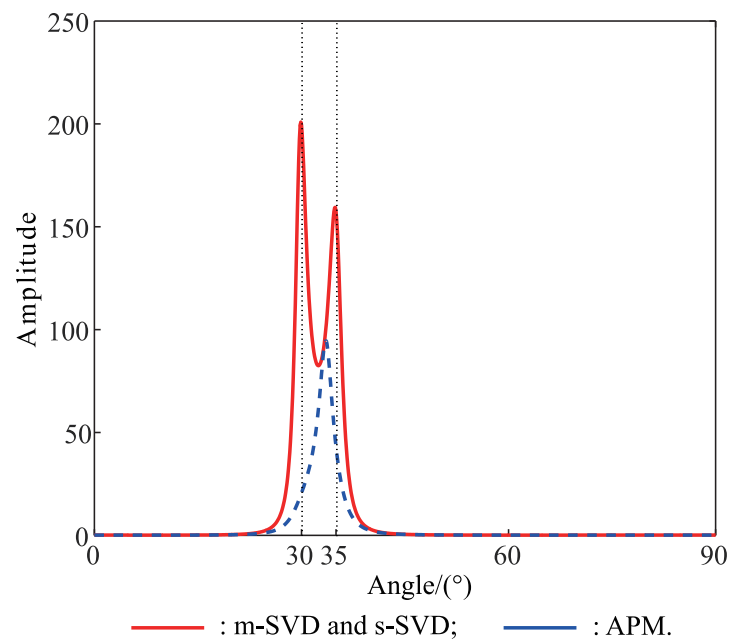

Fig. 3 An example of DOA estimation using MUSIC algorithm

Fig. 3 shows an example of DOA estimation using MUSIC algorithm with 10 sensors, $100 \mathrm{~s}$ apshots as $\mathrm{SNR}=$ $5 \mathrm{~dB}$, two close-spaced sources at $\theta_{1}=30^{\circ}$ and $\theta_{2}=34^{\circ}$. It can be seen clearly from Fig. 3 that for a low $\mathrm{SNR}=$ $5 \mathrm{~dB}$, the proposed methods can provide much sharper MUSIC spectrums than APM with $n=7$. The proposed methods can successfully resolve the two close-spaced sources while APM fails, and therefore, the new techniques are much more robust against the noise as compared to APM. Considering APM with $n=7$ has much higher complexity than the proposed algorithms, this example demonstrates again that there are significant advantages in complexity reduction by the proposed methods as compared to APM.

Finally, we compare the computational efficiencies of various methods including (7), (8), APM and the proposed m-SVD-, s-SVD- techniques in Table 2, where the computational efficiency is evaluated by CPU time by running the Matlab code in the same environment. In the simulation, we set $m=15, r_{1}=r_{2}=11$, and generate $\boldsymbol{\Psi}_{i} \in \mathbf{C}^{m \times r_{i}}(i=1,2)$ by using (27) and (28). The simulation times are plotted as functions of $n$ in terms of the averaging of 500 independent Monte Carlo trials

$$
\bar{t} \triangleq \frac{1}{500} \sum_{i=1}^{500} t_{i} .
$$

Table 2 Simulation time versus iteration number $n$

\begin{tabular}{ccccccc}
\hline Algorithm & $n=1$ & $n=5$ & $n=10$ & $n=15$ & $n=20$ & $n=25$ \\
\hline APM & 0.012 & 0.038 & 0.051 & 0.058 & 0.062 & 0.064 \\
Method in (7) & 0.021 & 0.022 & 0.023 & 0.021 & 0.022 & 0.022 \\
Method in (8) & 0.034 & 0.033 & 0.036 & 0.033 & 0.035 & 0.038 \\
m-SVD & 0.029 & 0.028 & 0.028 & 0.030 & 0.028 & 0.028 \\
s-SVD & 0.011 & 0.012 & 0.016 & 0.012 & 0.012 & 0.014 \\
\hline
\end{tabular}

It can be seen clearly from Table 2 that the proposed m-SVD- and s-SVD- methods cost much lower simulation time than the APM technique, especially with large $n$ 's. It is also seen from the table that our methods cost slightly smaller simulation time as the two algorithms in (7) and (8). Therefore, the proposed m-SVD- and s-SVD- methods are the two most efficient methods among the five ones. Considering that s-SVD is much more efficient than mSVD with the same accuracy, we suggest to use the former instead of the latter in practice.

\section{Conclusions}

We have proposed two new methods, m-SVD and s-SVD, for computing the intersection of two subspaces. Both the two techniques obtain the orthogonal basis directly with closed-forms and reduced-complexities as compared to APM. Further research should include extending the proposed methods to compute the intersection of more than two subspaces.

\section{References}

[1] NI Y, ZHAO J, WANG Y, et al. Beamforming and interference cancellation for D2D communication assisted by twoway decode-and-forward relay node. China Communications, 2018, 15(3): 100-111.

[2] ZHANG Y, HE P, PAN F. Three-dimension localization of wide-band sources using sensor network. Chinese Journal of Electronics, 2017, 26(6): 1302 - 1307.

[3] YAN F G, LIU S, WANG J, et al. Fast DOA estimation using co-prime array. Electronics Letters, 2018, 54(7): 409-410.

[4] YAO B, ZHANG W, WU Q. Weighted subspace fitting for twodimension DOA estimation in massive MIMO systems. IEEE Access, 2017, 5: 14020 - 14027.

[5] LI J, LI D, JIANG D, et al. Extended-aperture unitary root MUSIC-based DOA estimation for coprime array. IEEE Communications Letters, 2018, 22(4): $752-755$.

[6] TIAN X, LEI J, DU L. A generalized 2-D DOA estimation method based on low-rank matrix reconstruction. IEEE Access, 2018, 6: $17407-17414$.

[7] NAGANANDA K G, ANAND G V. Subspace intersection method of bearing estimation in shallow ocean using acoustic vector sensors. Proc. of the 16th European Signal Processing Conference, 2008: 1-5.

[8] ANAND G V, NAGESHA P V. High resolution bearing estimation in partially known ocean using short sensor arrays. Proc. of the International Symposium on Ocean Electronics, 2011: $47-56$.

[9] PANG J, LIN J, ZHANG J, et al. Subspace intersection method of bearing estimation based on least square approach in shallow ocean. Proc. of the IEEE International Conference on Acoustics, Speech and Signal Processing, 2008: 2433-2436.

[10] GESBERT D, VAN DER VEEN A J, PAULRAJ A. On the equivalence of blind equalizers based on MRE and subspace intersection. IEEE Trans. on Signal Processing, 1999, 47(3): $856-859$.

[11] BARBAROSSA S. Parameter estimation of multicomponent polynomial-phase signals by intersection of signal subspaces. Proc. of the 8th Workshop on Statistical Signal and Array Processing, 1996: 452-455.

[12] PETROCHILOS N, VAN DER VEEN A J. Blind time delay estimation in asynchronous CDMA via subspace intersection 
and ESPRIT. Proc. of the IEEE International Conference on Acoustics, Speech and Signal Processing, 2001, 4: $2217-$ 2220.

[13] MURDOCK C, TORRE F D L. Approximate grassmannian intersections: subspace-valued subspace learning. Proc. of the IEEE International Conference on Computer Vision, 2017: $4318-4326$.

[14] AL-SAEDY M, AL-IMARI M, AL-SHURAIFI M, et al. Joint user selection and multimode scheduling in multicell MIMO cellular networks. IEEE Trans. on Vehicular Technology, 2017, 66(12): $10962-10972$.

[15] YUAN B, LIAO X, GAO F, et al. Achievable degrees of freedom of the four-user MIMO Y channel. IEEE Communications Letters, 2014, 18(1): 6-9.

[16] MARKOVIĆ D, ANTONACCI F, SARTI A, et al. 3D beam tracing based on visibility lookup for interactive acoustic modeling. IEEE Trans. on Visualization and Computer Graphics, 2016, 22(10): $2262-2274$.

[17] GOLUB G H, VAN LOAN C H. Matirx computations. Baltimore, MD, USA: The Johns Hopkins University Press, 1996.

[18] ANDERSON JR W N, DUFFIN R J. Series and parallel addition of matrices. Journal on Mathematics Analysis \& Applications, 1968, 26(3): 576-594.

[19] VON NEUMANN J. Functional operators II: the geometry of orthogonal spaces. Princeton: Princeton University Press, 1950.

[20] ARONSZAJN N. Theory of reproducing kernels. Transactions of the American Mathematics Society, 1950, 68(3): 337-404.

[21] KAYALAR S, WEINERT H. Error bounds for the method of alternating projections. Mathematics of Control Signals \& Systems, 1988, 1(1): $43-59$.

[22] DEUTSCH F. The angle between subspaces of a Hilbert space. Singh S P ed. Approximation theory, wavelets and applications. Dordrecht: Springer Netherlands, 1995: 107-130.

[23] DEUTSCH F. Best approximation in inner product spaces. New York: Springer, 2001.

[24] HALPERIN I. The product of projection operators. Acta Scientiarum Mathematicarum, 1962, 23(23): 96-99.

[25] BEN-ISRAEL A. Projectors on intersection of subspaces. benisrael.net/BEN-ISRAEL-NOV-30-13.pdf.

[26] ZHOU M, VAN DER VEEN A J. Improved subspace intersection based on signed URV decomposition. Proc. of the Asilomar Conference on Signals, Systems, and Computers, 2011: $2174-2178$.

[27] ZHANG Z, LENG W, WANG A, et al. Effective estimation of the desired-signal subspace and its application to robust adaptive beamforming. Proc. of the IEEE International Conference on Acoustics, Speech and Signal Processing, 2017: $3370-$ 3374.

[28] PHAN H M. Linear convergence of the douglas-rachford method for two closed sets. Optimization, 2016, 65(2): $369-$ 385.

[29] HESSE R, LUKE D R. Nonconvex notions of regularity and convergence of fundamental algorithms for feasibility problems. SIAM Journal on Optimization, 2013, 23(4): $2397-$ 2419.

[30] DAVIS D, YIN W. Faster convergence rates of relaxed Peaceman-Rachford and ADMM under regularity assumptions. Mathematics of Operations Research, 2014, 42(3): $1-$ 40.

[31] GISELSSON P, BOYD S. Linear convergence and metric selection for Douglas-Rachford splitting and ADMM. IEEE Trans. on Automatic Control, 2017, 62: 532-544.

[32] GISELSSON P. Tight global linear convergence rate bounds for Douglas-Rachford splitting. Journal of Fixed Point Theory and Applications, 2017, 19(4): $2241-2270$.

[33] BAUSCHKE H H, CRUZ J Y B, NGHIA T T A, et al. The rate of linear convergence of the Douglas-Rachford algorithm for subspaces is the cosine of the Friedrichs angle. Journal of Approximation Theory, 2014, 185: 63-79.

[34] DOUGLAS J, RACHFORD H H. On the numerical solution of heat conduction problems in two and three space variables. Transactions of the American Mathematical Society, 1956, 82(2): $421-439$.

[35] FÄLT M, GISELSSON P. Optimal convergence rates for generalized alternating projections. Proc. of the 56th IEEE Annual Conference on Decision and Control, 2017: 2268 - 2274.

[36] BAUSCHKE H H, CRUZ J Y B, NGHIA T T A, et al. Optimal rates of linear convergence of relaxed alternating projections and generalized Douglas-Rachford methods for two subspaces. Numerical Algorithms, 2016, 73(1): 33-76.

\section{Biographies}

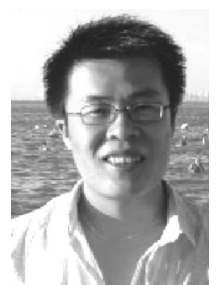

YAN Fenggang was born in 1982. He received his $\mathrm{Ph} . \mathrm{D}$. degree in information and communication engineering from Harbin Institute of Technology, Harbin, in 2014. From July 2008 to March 2011, he was a research associate of the Fifth Research Institute of China Aerospace Science and Technology Corporation (CASC), where his research mainly focused on the processing of remote sensing images. Since October 2015, he became an associate professor of the Department of Electronics Information Engineering, Harbin Institute of Technology at Weihai, Weihai, China. His current research interests include array signal processing and statistical performance.

E-mail: yfglion@163.com

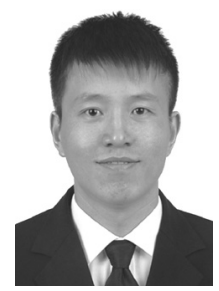

LIU Shuai was born in 1980. He received his B.E. and M.S. degrees from Northwestern Polytechnical University China, in 2002 and 2005, respectively, and received his Ph.D. degree in information and communication engineering from Harbin Institute of Technology, China, in 2013. Since 2013, he has been an associate professor of the School of Information and Electricity Engineering, Harbin Institute of Technology, Weihai, China. His current interests are in the area of conformal array and polarization sensitive array signal processing.

E-mail: liu_shuai_boy@163.com

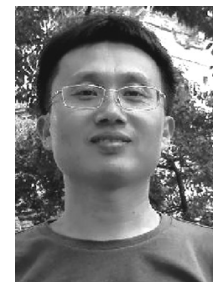

WANG Jun was born in 1976. He received his Ph.D. degree in information and communication engineering from Harbin Institute of Technology, Harbin, China, in 2014. Since 2015, he has been an associate professor of the Department of Electronics information Engineering, Harbin Institute of Technology at Weihai, Weihai, China. His current research interest is mainly radar signal processing. E-mail: hitwangjun@126.com

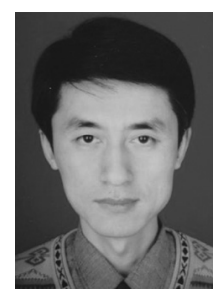

JIN Ming was born in 1968. He received his B.E., M.S., and Ph.D. degrees in information and communication engineering from Harbin Institute of Technology, China, in 1990, 1998 and 2004, respectively. From 1998 to 2004, He was with the Department of Electronics Information Engineering, Harbin Institute of Technology. Since 2006, he became a professor of the School of Information and Electricity Engineering, Harbin Institute of Technology at Weihai. His current interests include array.

E-mail: jinming0987@163.com 